\title{
Chrysanthemum latifolium $x$ C. grandiflorum cv. 'Red Betty' crossed to induce new cultivars: Hybrid genome characterization and species relationship analyzed by FISH and GISH
}

\author{
Magdy Hussein Abd El-Twab ${ }^{1}$ and Katsuhiko Kondo ${ }^{2,3}$
${ }^{1}$ Department of Botany and Microbiology, Faculty of Science, Minia University, El Minia City 61519, Egypt;
${ }^{2}$ Laboratory of Plant Genetics and Breeding Science, Department of Agriculture,
Faculty of Agriculture, Tokyo University of Agriculture, 1737 Funako, Atsugi City, Kanagawa Prefecture, 243-0034, Japan

\author{
${ }^{3}$ Author for correspondence: k3kondo@nodai.ac.jp \\ Received January 1, 2014; acceptd February 11, 2014
}

\begin{abstract}
Hybridity, genome differentiation and relationship were investigated in an interspecific $\mathrm{F}_{1}$ hybrid $(2 \mathrm{n}=45)$ artificially produced between Chrysanthemum latifolium (Shih) H. Ohashi et Yonek. (formerly: Ajania latifolia Shih) (2n=36) and C. grandiflorum Ramat cv. 'Red Betty' (2n=54) by FISH (fluorescent in situ hybridization) and GISH (genomic in situ hybridization) using 5S, 45S and total genomic DNA labeled probes. Seventy-three hybrid seedlings were successfully produced only in vitro. FISH on the chromosomes of the hybrid had 11 signals of $45 \mathrm{~S}$ rDNA sites, while five and six signals of the $5 \mathrm{~S}$ rDNA were detected. GISH distinguished approximately 27 -yellow-colored chromosomes were hybridized with the probe of $C$. latifolium and 18 redcolored were not, which could be characteristics of C. grandifforum cv. 'Red Betty'. Nine out of the 27 chromosomes were common homologous chromosomes of the two genomes. The 18 red-colored chromosomes, which isolated by GISH without using blocking DNA, might come from a distantly related ancestor of $C$. latifolium.
\end{abstract}

KEYWORDS: Chrysanthemum latifolium, Chrysanthemum grandiflorum cv. 'Red Betty', 5S rDNA, 45S rDNA, Fluorescent in situ hybridization, Genomic in situ hybridization

Chrysanthemum grandiflorum (Ramat.) Kitamura is considered one of the most important and commonest ornamental plants of the world. Traditionally, useful agronomical traits have been introduced into the species by conventional cross-breeding and mutation breeding (eg. Preil et al. 1983; De Jong and Radmaker 1986; Dalsou and Short 1987; Huitema et al. 1987). Plants that crosspollinate Chrysanthemum can be used for conventional cross-breeding with this species. Wide hybridizations between the garden Chrysanthemum and some of the wild relatives have been attempted on a significant scale in China and Japan (Chen et al. 1995; Furuta et al. 2004). Hexaploidy of Chrysanthemum cultivars (hexaploidy) play an important role in chromosome characterization and breeding of the plants (Dowrick 1953; Abd El-Twab and Kondo 2004a). Thus, genome characterization of closely related taxa is an important to identify ancestors, which might contribute to satisfactory phylogenetic and breeding cultivars (Leich and Bennett 1997; Soltis and Soltis 2000).

Fluorescence in situ hybridization (FISH) is an exceptionally important tool for chromosome physical localization of the 18S-5.8S-25S ribosomal RNA genes (45S rDNA) and 5S ribosomal RNA (5S rDNA), which has provided a group of valuable chromosome pointer markers. The chromosomal localization of the $45 \mathrm{~S}$ and $5 \mathrm{~S}$ rDNA via FISH in the chromosome complements of several wild species of Chrysanthemum and cultivars were reported (eg. Kondo and Abd El-Twab 2002; Abd El-Twab and Kondo 1999, 2003).

The technique of genomic in situ hybridization (GISH) has been useful for recognition of parental genomes and resolve levels and merging positions of alien chromatin, in interspecific and intergeneric plant hybrids after using total genomic DNA of one parents (e.g., Bennett et al. 1992; Chen et al. 1995; Takahashi et al. 1997; Kondo and Abd El-Twab 2002). GISH was applied on the chromosomes of several combinations of artificial hybrids in Chrysanthemum (eg. Kondo et al. 1999; Kondo and Abd El-Twab 2002; Abd El-Twab et al. 1999; Abd ElTwab and Kondo 1999, 2000, 2001a, b, 2004a,b, 2006, 2007, 2008).

In our laboratory special interest has been directed toward group of cultivars in C. grandiflorum and closely related wild species that possess agronomical useful characters for breeding such as small leaves, favorable odors and disease resistance. Therefore, the aims of this study are to: 1) investigate the cross ability between the two species; 2) detected the physical localization of the $5 \mathrm{~S}$ and $45 \mathrm{~S}$ rDNA sites through simultaneous use of $5 \mathrm{~S}$ and 45S rDNA FISH probes; 3) explore genetic relationships by GISH in the genome of the artificial hybrid.

\section{Materials and Methods}

Materials The plants used in this study were the maternal Chrysanthemum latifolium (Shih) H. Ohashi et Yonek. (formely: Ajania latifolia Shih $(2 \mathrm{n}=36)$ and the paternal C. grandiflorum (Ramat.) Kitamura cv. 'Red Betty' $(2 n=54)$. The plant species and hybrids have been preserved in the Laboratory of Plant Genetics and Breeding Science, Department of Agriculture, Faculty of Agriculture, Tokyo University of Agriculture, Funako 1737, Atsugi City, Kanagawa Prefecture, Japan. 
Cross hybridization method The taxa were utilized for artificial hybridization by hand pollination, followed Tanaka et al. (1989) and Abd El-Twab and Kondo (2000). Subsequently fertilized embryos were cultured in vitro following Abd El-Twab and Kondo (2006).

Chromosome preparations Growing root tips were collected from one-year-old seedlings (A-1-10-1, 2, 3) and were pretreated, fixed, macerated, spread, stained and observed according to Kondo et al. (1996).

Ribosomal DNA probes 5 S rDNA probe The 5S rDNA probe was produced by the polymerase chain reaction (PCR) method as described by Abd El-Twab and Kondo (2002).

$45 S$ rDNA probe The probe of pTa71 (45S rDNA) consisted of a $9 \mathrm{~kb}$ Eco RI fragment of rDNA derived from Triticum aestivum L. (Gerlach and Bedbrook 1979), which was recloned into pUC19 plasmid. It was comprised of the coding sequence for the $18 \mathrm{~S}, 5.8 \mathrm{~S}$ and $26 \mathrm{~S}$ genes and the non-transcribed spacer sequences.

Total genomic DNA extraction DNA was isolated from 0.5-1.0 $\mathrm{g}$ of fresh young leaf material of the two parental species following the protocol of Kondo et al. (1999). Total genomic DNA of Chrysanthemum latifolium and $C$. grandiflorum cv. 'Red Betty' were mechanically sheared and labeling was carried out according to the manufacturer's protocol.

Labeling of the probes The probes were labeled either with biotin-14-dATP nick translation kit (Gibco BRL) or digoxigenin (DIG)-dUTP by Dig DNA labeling kit (Boehringer Mannheim) labeling of each probe was carried out according to the manufacturer's protocols.

The procedure of FISH and GISH The probe hybridization mixture $(20 \mu \mathrm{l}$ per slide) contained about $50 \mathrm{ng}$ of each DNA labelled probe, $10 \%$ dextran sulphate, $2 \mathrm{X}$ SSC, $0.25 \%$ SDS (lauryl sulphate). The probe mixtures were denatured by boiling for $5 \mathrm{~min}$, centrifuged briefly (Abd El-Twab and Kondo 2004).

Detection and visualization of the hybridized probes followed Abd El-Twab et al. (2004) For double probes multicolor FISH, the yellow-color was visualized by FITC after the hybridization with the biotin labeled probes and red-color was visualized by antidigoxigenin rhodamin and the counter stain was blue of DAPI, while the counter stain was red color of PI for single probe. The fluorescence signals were examined with an epifluorescence microscope with Nikon B-2A filter cassette (yellow color), R filter (red color), UV filter (blue color) and microphotographs were taken on CCD camera (Pixera Pengium 600CL). Analysis of hybridization signals and superimposed images were produced using Adobe Photoshop 7.

\section{Results and Discussion}

Hybrids were successfully produced after 684 florets of 40 heads of the maternal Chrysanthemum latifolium were pollinated artificially by C. grandiflorum cv. 'Red Betty', and produced $483(71 \%)$ fertile and 101 (14.8\%) aborted achenes. Seventy-three (25.8\%) seedlings were germinated out of 283 achenes were sown in vitro, while no germination in vivo.

Many cross combinations have proven to be nonfeasible, for a number of reasons, including disharmony between the parental genomes when the parents of the wide cross differ in their ploidy (Wolff and Rijin 1993) and the failure of hybrid embryos to develop beyond an early stage (Tanaka and Watanabe 1972). The ability to overcome sexual incompatibility has been improved by the elaboration of in vitro ovary and embryo culture (Watanabe 1977), and these techniques have been applied to obtain a number of wide hybrids involving various Chrysanthemum species (Kondo et al. 1999; Abd El-Twab and Kondo 2004b, 2006). In the present study only germination $(25.8 \%$ ) were detected in vitro, while no germination was in vivo indicating that embryo rescue might be necessary to produce high rate of germination for the present hybrid combination. The hybrids showed expected chromosome number of $2 \mathrm{n}=45$ (Fig. 1). The genome constitution of the hybrid and fate of the parental chromosomes were studied in mitotic metaphase chromosomes of the $\mathrm{F}_{1}$ hybrid by FISH using $45 \mathrm{~S}$ and $5 \mathrm{~S}$ rDNA genes and GISH using labeled total genomic DNA probe of one of the parents.

FISH multicolor was applied on the mitotic chromosomes of the hybrid, using the digoxiginin labeled pTa71 of $45 \mathrm{~S}$ rDNA and the biotin labeled 5S rDNA probes. FISH showed 11 red color signals of $45 \mathrm{~S}$ rDNA sites, while five and six yellow green signals of the $5 \mathrm{~S}$ rDNA (Fig. 2A, C-F). In Chrysanthemum the 5S rDNA arrays tend to be localized at one site on one to three homologous pairs of chromosomes (Abd El-Twab and Kondo 2002). Use of double- or multiple-probe FISH facilitates the identification of homologous chromosomes and the difference of changes in genome structure among species. It also helps to clarify genetic maps and to assign linkage groups to physically marked chromosomes. The present FISH linkage linkage patterns of the 45S and 5S rDNA is the Chrysanthemum type (Abd El-Twab and Kondo 2012), which revealed that the chromosomes harbored loci of rDNA distributed separately on different chromosomes.

GISH was applied on somatic chromosomes of meristematic root tips that were pretreated in 8-hydroxyquenoline (Fig. 2B). GISH distinguished approximately 27 yellow-colored chromosomes (instead of 18 chromosomes) were hybridized with the biotin labeled probe of $C$. latifolium (yellow color of FITC), which might indicate the nine chromosomes were common between the two genomes, while the other 18 red-colored chromosomes were not hybridized and 


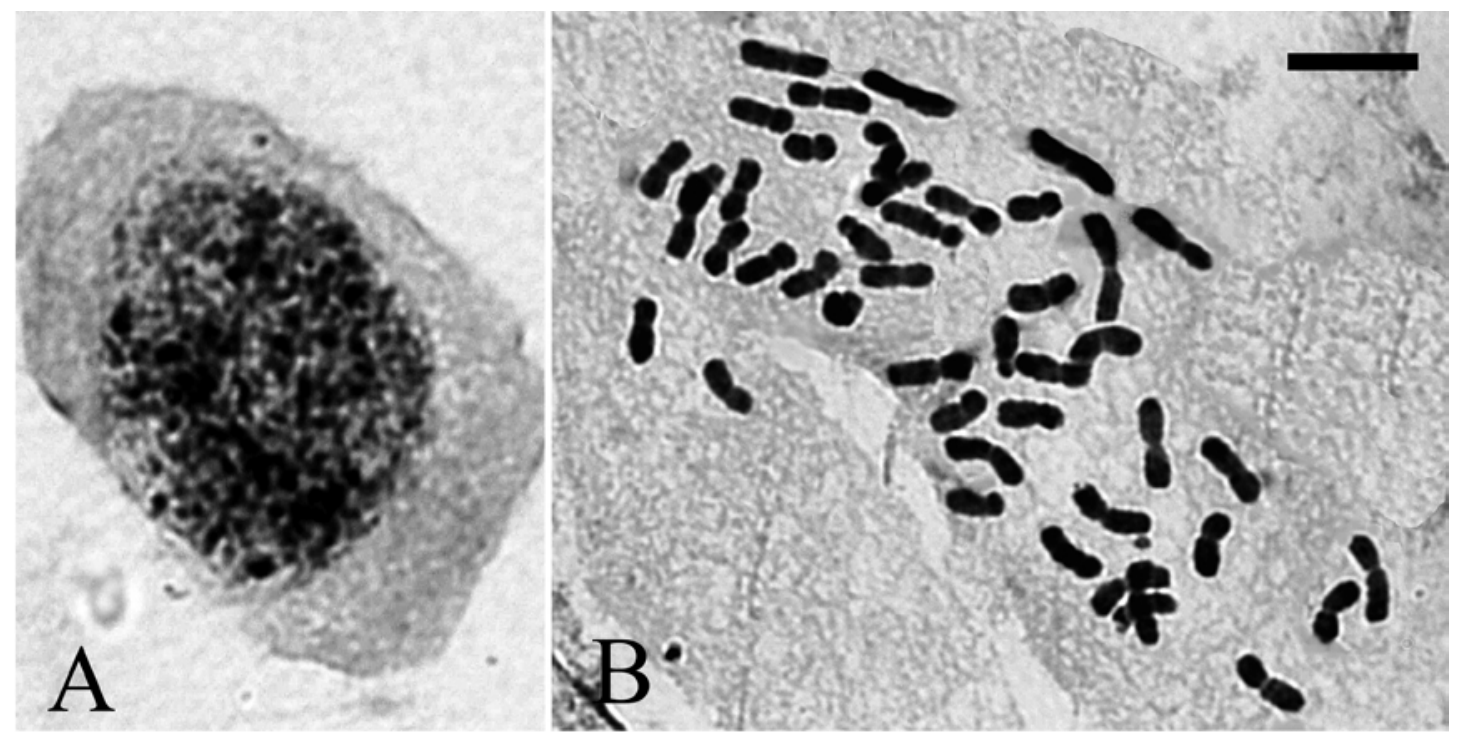

Fig. 1. Somatic chromosomes in the artificial interspecific $F_{1}$ hybrid $(2 n=45$, pentaploid) between Chrysanthemum latifolium and C. grandiflorum cv 'Red Betty'. A. Resting chromosome. B. Metaphase chromosomes $2 \mathrm{n}=45$ (pentaploid) Bar $=10 \mu \mathrm{m}$.

thus could be characteristics of C. grandiflorum cv. 'Red Betty' (red color of PI).

Distribution of repetitive DNA sequences should be clarified to gain insight into the organization, evolution and behavior of plant genomes. A particular repetitive sequence may be present in many species within a taxonomic family, or be essentially species specific (Harrison and Heslop-Harrison 1995). The distribution of a repetitive sequence can indicate mechanisms and constraints on both genome and species evolution in Chrysanthemums (Abd El-Twab and Kondo 2012). The intensity of fluorescence in GISH preparations reflects sequence homology between the total genomic DNA probe and the target, and thus can be used as a measure of homology between distinct genomes (Kondo et al. 1999; Abd El-Twab and Kondo 2001a; Kondo and Abd El-Twab 2002). GISH analysis, when a hybrid is easily obtained, large excess of blocking DNA is necessary to achieve genome discrimination in the hybrid (Abd El-Twab et al. 1999; Kondo and Abd El-Twab 2002), while hybrids (which sometimes require embryo rescue for their success), which no blocking DNA is needed to fully discriminate between the parental genomes that suggests sequence homology is less close (Kondo et al. 1999; Abd El-Twab and Kondo 2001b, 2004, 2006). Therefore, the ease with which GISH could discriminate between parental genomes the distant genetic relationship to one another (and vice versa). The present results indicates that approximately 27 chromosomes with yellow color have nine common chromosomes between the two genomes, while the other 18 chromosomes are characteristics and related to C. grandiflorum in particular to the cultivar 'Red Betty' that used for producing the artificial cross hybrids. The interspecific cross-ability in Chrysanthemum, cytological analysis and molecular cytogenetics evaluation of the artificial produced hybrids will enable us to understand what can be done in taxa breeding and suggested that interspecific hybridization between wild Chrysanthemum species and cultivars is an effective way for new strain improvement.

ACKNOWLEDGEMENTS. This study was supported by the Grantin-Aid for Scientific Research Program (A)(1) No. 19255004 (ID: L09550) and was supported by the long-term visiting researcher (ID: L09550) from April 2009 to February 2010 by Japan Society for the Promotion of Science to Tokyo University of Agriculture under the directorship by Katsuhiko Kondo.

\section{Literature Cited}

Abd El-Twab, M. H. and Kondo, K. 2000. Discrimination and isolation of terminal chromosomal regions of Dendranthema occidentali-japonense in the chromosomes of $F_{1}$ hybrid of Dendranthema boreale by using GISH. Chrom. Sci. 4: 87-93.

Abd El-Twab, M. H. and Kondo, K. 2002. Physical mapping of 5S rDNA in chromosomes of Dendranthema by fluorescence in situ hybridization. Chrom. Sci.6: 13-16.

Abd El-Twab, M. H. and Kondo, K. 2003. Physical mapping of 45S rDNA loci by fluorescent in situ hybridization and Evolution among polyploid Dendranthema species. Chrom. Sci.7: 71-76.

Abd El-Twab, M. H. and Kondo, K. 2004a. Identification of mutation and homologous chromosomes in four cultivars of Dendranthema grandiflora by physical mapping of $5 \mathrm{~S}$ and $45 \mathrm{~S}$ rDNA using fluorescence genomic in situ hybridization. Chrom. Sci. 8: 81-86.

Abd El-Twab, M. H. and Kondo, K. 2004b. Identification of parental chromosomes and changes of artificial, intergeneric $\mathrm{F}_{1}$ hybrid between Dendranthema horaimontana and Nipponanthemum nipponicum by fluorescence genomic in situ hybridization (FISH) and fluorescence in situ hybridization (GISH). Chrom. Sci. 8: 71-79.

Abd El-Twab, M. H. and Kondo, K. 2006. Fluorescence in situ hybridization and genomic in situ hybridization to identify the parental genomes in the intergeneric hybrid between Chrysanthemum japonicum and Nipponanthemum nipponicum. Chrom. Bot. 1: 7-11.

Abd El-Twab, M. H. and Kondo, K. 2007. Rapid genome 


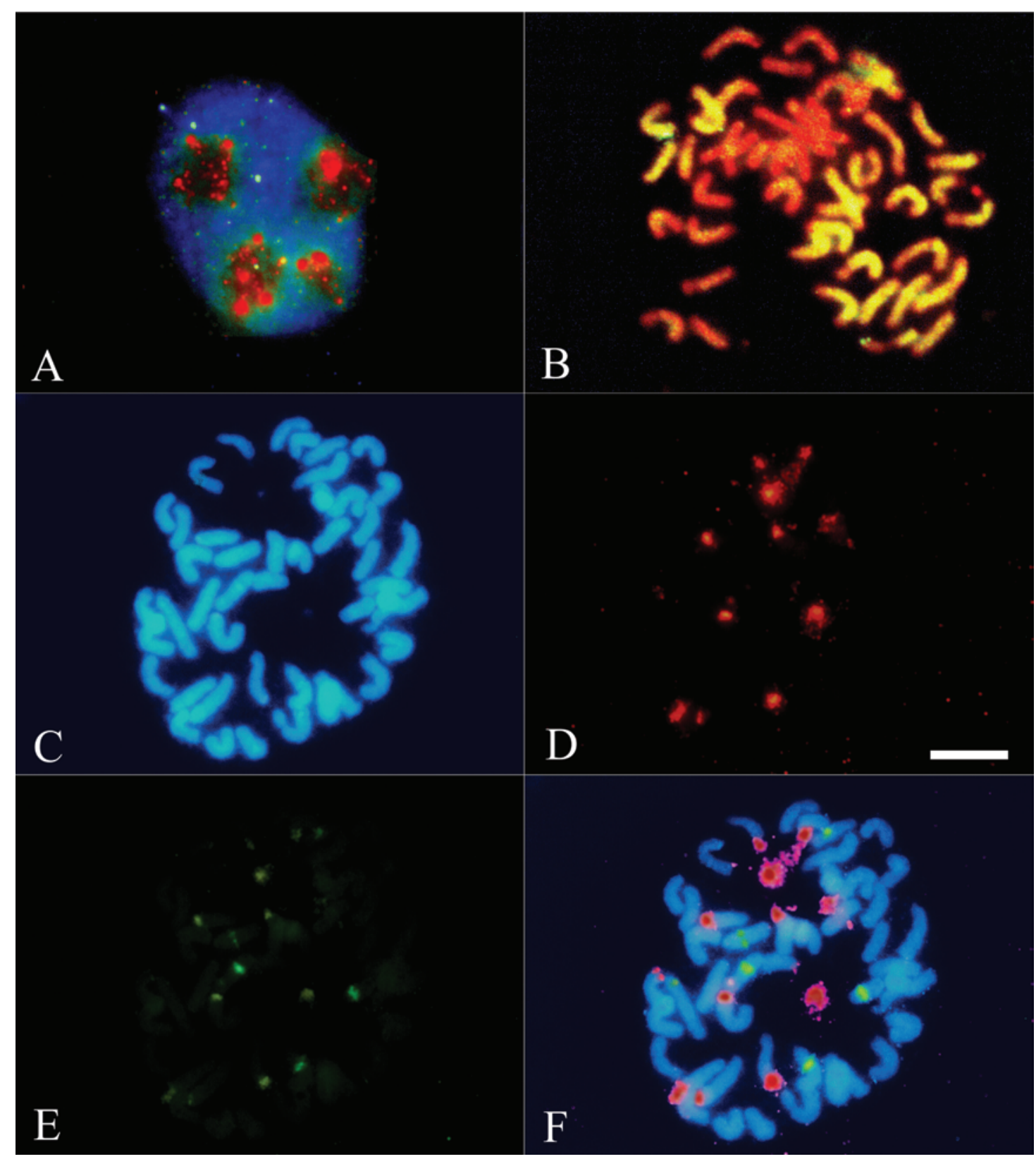

Fig. 2. Simultaneous FISH (A, C-F) and GISH (B) applied on mitotic chromosomes in the artificial interspecific $\mathrm{F}_{1}$ hybrid ( $2 \mathrm{n}=45$, pentaploid) between Chrysanthemum latifolium and $C$. grandiflorum $\mathrm{cv}$ 'Red Betty'. Biotin labeled total genomic DNA probe of $C$. latifolium (B; sn: A-1-10-1) were used. Digoxiginine of the 45S rDNA and biotin-labeled 5S rDNA probes were applied (A, C-F, antidigoxiginine Rhodamin red color, avidin-FITC yellow and blue color of DAPI counter stain). Yellow-color was fluoresced and visualized by FITC and red-color by PI (B). B. Chromosome separation after using the probe bright green-yellow signals on approxematly 27 chromosomes hybridized with biotin labeled probe of $C$. latifolium and 18 red colored chromosomes were not hybridized. A. Yellow-green colored interphase nucleus was hybridized with the probe showing big four red color signals of the 45S rDNA in four nucleoli and small yellow green signals of $5 \mathrm{~S}$ rDNA. 11 red signals of the $45 \mathrm{~S} \mathrm{rDNA}$ (D and F) and 5 yellow of $5 \mathrm{~S}$ rDNA (E and F) were observed on the superimposed image of the metaphase chromosomes. $\mathrm{Bar}=10 \mu \mathrm{m}$.

reshuffling induced by allopolyploidy in $\mathrm{F}_{1}$ hybrid in Chrysanthemum sensu lato (Ajania-Dendranthema). Chrom. Bot. 2: 1-9.

Abd El-Twab, M. H. and Kondo, K. 2008. Visualization of genomic relationships in allotetraploid hybrids between Chrysanthemum lavandulifolium X Ch. chanetii by fluorescence in situ hybridization and genomic in situ hybridizarion. Chrom. Bot. 3: 19-25.

Abd El-Twab, M. H. and Kondo, K. 2012. FISH Physical linkage of $5 \mathrm{~S}$ and $45 \mathrm{~S}$ rDNA in Chrysanthemum in broad sense (Anthemideae) and species relationships. Journal of Genetics 91: 245-249.

Abd El-Twab, M. H. and Kondo, K. 2001a. Genome territories of Dendranthema horaimontana in mitotic nuclei of $F_{1}$ hybrid between Dendranthema horaimontana and Tanacetum parthenium. Chrom. Sci. 5: 63-71.

Abd El-Twab, M. H. and Kondo, K. 2001b. Molecular cytogenetic identification of the parental genomes in the intergeneric hybrid between Leucanthemella linearis and Nipponanthemum nipponicum during meiosis and 
mitosis. Caryologia 54: 109-114.

Abd El-Twab, M. H., and Kondo, K. 1999. Identification of nucleolar organizing regions and parental chromosomes in $\mathrm{F}_{1}$ hybrid of Dendranthema japonica and Tanacetum vulgare simultaneously by fluorescence in situ hybridization. Chrom. Sci. 3: 59-62.

Abd El-Twab, M. H., Kondo, K. and Hong, D. 1999. Isolation of a particular chromosome of Ajania remotipinna in a chromosome complement of an artificial $\mathrm{F}_{1}$ hybrid of Dendranthema lavandulifolia X Ajania remotipinna by use of genomic in situ hybridization. Chrom. Sci. 3: 2128.

Abd El-Twab, M. H., Shinoyama, H. and Kondo, K. 2004. Evidences of intergeneric somatic-hybrids between Dendranthema grandiflora cv. Shuho-no-chikara and Artemisia sieversiana and their chromosomal mutations by using fluorescence in situ hybridization and genomic in situ hybridization. Chrom. Sci. 8: 29-34.

Bennett, S. T., Kenton, A. Y. and Bennett, M. D. 1992. Genomic in situ hybridization reveals the allopolyploid nature of Milium montanum (Gramineae). Chromosoma 101: 420-424.

Chen, Q., Conner, R. L. and Laroche, A. 1995. Identification of the parental chromosomes of the wheat-alien amphidiploid Agrotana by genomic in situ hybridization. Genome 38: 1163-1169.

Dalsou, V. and Short, K. C. 1987. Selection for sodium chloride tolerance in Chrysanthemums, Acta Hort. 212: 737-740.

De Jong, J. and Radmaker, W. 1986. The reaction of Chrysanthemum cultivars to Puccinia horiana and the inheritance of resistance. Euphytica 35: 945-952.

Dowrick, G. J. 1953. The chromosomes of Chrysanthemum. II. Garden varieties. Heredity 7: 59-72.

Furuta, H., Shinoyam, H., Nomur, Y., Maed, M. and Makara, K. 2004. Production of intergeneric somatic hybrids of Chrysanthemum $[$ Dendranthema $\times$ grandiflorum (Ramat.) Kitamura] and wormwood (Artemisia sieversiana J. F. Ehrh. ex. Willd) with rust (Puccinia horiana Henning) resistance by electrofusion of protoplasts. Pl. Sci. 166: 695-702.

Gerlach W. L. and Bedbrook J. R. 1979 Cloning and characterization of ribosomal RNA genes from wheat and barley. Nucl. Acid. Res. 7: 1869-1885.

Harrison, G. E. and Heslop-Harrison, J. S. 1995. Centromeric repetitive DNA sequences in the genus Brassica. Theoretical Applied Genettics 90: 157-165.

Huitema, J. B. M., Gussennoven, G. C., De Jong, J. and Dons, J. J. M. 1987. Selec-tion and in vitro characterization of low-temperature tolerant mutants of Chrysanthemum morifolium Ramat. Acta Hort. 197: 89-96.

Kondo, K. and Abd El-Twab, M. H. 2002. Analysis of intera- generic relationships Sensu stricto among the members of Chrysanthemum sensu lato by using fluorescence in situ hybridization and genomic in situ hybridization. Chrom. Sci. 6: 87-100.

Kondo, K., Abd El-Twab M. H. and Tanaka, R. 1999. Fluorescence in situ hybridization identifies reciprocal translocation of somatic chromosomes and origin of extra chromosome by an artificial, intergeneric hybrid between Dendranthema japonica and Tanacetum vulgare. Chrom. Sci. 3: 15-19.

Kondo, K., Abd El-Twab, M. H., and Tanaka, R. 1999. Fluorescence in situ hybridization identifies reciprocal translocation of somatic chromosomes and origin of extra chromosome by an artificial, intergeneric hybrid between Dendranthema japonica X Tanacetum vulgare. Chrom. Sci. 3: 15-19.

Kondo, K., Honda, Y., and Tanaka, R. 1996. Chromosome marking in Dendranthema japonica var. wakasaense and its closely related species by fluorescence in situ hybridization using rDNA probe. La Kromosomo II-81: 2785-2791.

Leich, I. J. and Bennett, M. D. 1997. Polyploidy in angiosperms. Trends in plant science, 2, 12:470-471.

Preil, W., Engelhardt, M. and Walther, F. 1983. Breeding of low temperature tolerant poinsettia (Euphorbia pulcherrima) and Chrysanthemum by means of mutation induction in in vitro culture. Acta Hort. 131: 345-351.

Soltis, P. S. and Soltis, D. E. 2000. The role of genetic and genomic attributes in the success of polyploids. Proc. Natl. Acad. Sci. USA 97: 7051-7057.

Takahashi, C., Leitch, I. J., Ryan, A., Bennett, M. D. and Brandham, P. E. 1997. The use of genomic in situ hybridization (GISH) to show transmission of recombinant chromosomes by a partially fertile bigeneric hybrid, Gasteria lutzii X Aloe aristata (Aloaceae), to its progeny. Chromosoma 105: 342-348.

Tanaka, R., Kawasaki, S., Yonezawa, Y., Taniguchi, K., and Ikeda, H. 1989. Cytogenetic studies on wild Chrysanthemum from China V. F-hybrids of Chrysanthemum lavandulifolium var. sianense $\mathrm{X} C h$. boreale. Cytologia 54: 365-372.

Tanaka, R. and Watanabe, K. 1972. Embryological studies in Chrysanthemum makinoi and its hybrid crossed with hexaploid Ch. japonense. Journal of Science, Hiroshima University, Series B, Division 2, 14: 75-84.

Watanabe, K. 1977b. Successful ovary culture and production of $\mathrm{F}_{1}$ hybrids and androgenic haploids in Japanese Chrysanthemum species. Journal of Heredity 68: 317320.

Wolff, K. and Rijn, J. P. 1993. Rapid detection of genetic variability in Chrysanthemum (Dendranthema grandiflora Tzvelev) using random primers. Heredity 71: 335-341. 
